\title{
System evaluation for a decision support system
}

\begin{abstract}
System evaluation is a necessary step in system development process to measure the successfulness of a system. However, this step has often been overlooked by system developers during the development process. This study aimed to discuss several system evaluations for Decision Support Systems (DSSs) and to explain the methodology used to evaluate a DSS model. In this study, a DSS model has been developed to assist decision makers to select an appropriate tree species to be planted for commercial tree planting. Based on few literatures, eight usability factors (efficiency, understandability, operability, attractiveness, error prevention, learnability, accuracy and effectiveness) have been identified for the evaluation process. The results present the usability level for each factor and indicated the tested DSS model is in the excellent level. It is anticipated that system developers can improve the DSS based on these findings as well as from the comments and suggestions made by the respondents.
\end{abstract}

Keyword: System evaluation; Decision support system; Usability testing 